\title{
Estudio Antropométrico en Párvulos Atendidos por el Sistema Educativo Público Chileno para el Diseño de Mobiliario
}

\author{
Anthropometric Study of Infants in the \\ Chilean Public Education System for Furniture Design
}

\author{
Jimena Rojas Colvin*; Atilio Aldo Almagià Flores** \& Juan Sebastián Ilardi***
}

\begin{abstract}
ROJAS, C. J.; ALMAGIÀ, F. A. A. \& ILARDI, J. S. Estudio antropométrico en párvulos atendidos por el sistema educativo público chileno para el diseño de mobiliario. Int. J. Morphol., 31(1):189-196, 2013.

RESUMEN: Para actualizar el diseño de mobiliario que usan los niños de los Jardines Infantiles del sistema público chileno desde 1970, se realiza un Estudio Ergonómico que permita determinar criterios y dimensiones para proponer un estándar para el diseño de un nuevo mobiliario. Este trabajo presenta los resultados del levantamiento antropométrico realizado a 2338 niños y niñas desde los 3 meses a 6 años de edad, en jardines de la Junta Nacional de Jardines Infantiles (JUNJI) en las regiones I, IX, X, XII y Metropolitana. Si bien un estudio de las variables de peso y talla registradas en el Sistema de Gestión de Información de la Institución para niños y niñas de Jardines Clásicos urbanos manifestaba que no habían diferencias significativas entre zonas geográficas, se seleccionaron regiones considerando diversidad intercultural por ancestros directos de origen extranjero y/o de pueblos originarios en caso que la proporcionalidad de los segmentos manifestara diferencias, aún cuando la estatura y peso fueran similares. Se midieron 19 parámetros antropométricos para el desarrollo de mobiliario preescolar con criterios ergonómicos estandarizados al que se agregó una variable para orientar el ancho de uso de una superficie de trabajo. Los resultados obtenidos de la muestra considerada, nos permiten afirmar que se requiere hacer modificaciones en las dimensiones del mobiliario en uso. Se identificó una discrepancia importante entre las dimensiones antropométricas de los niños y el mobiliario existente. Se proponen tablas antropométricas para niños y niñas desde los 3 a 72 meses de edad, que indicarían que el mobiliario en uso no es del tamaño correcto para lograr comodidad y bienestar.
\end{abstract}

PALABRAS CLAVE: Párvulos; Antropometría; Ergonomía; Mobiliario Preescolar.

\section{INTRODUCCIÓN}

Para el diseño de productos es necesario conocer las dimensiones corporales de los usuarios (Ávila Chaurand et al., 2001). En el caso de los infantes, estas consideraciones son relevantes para evitar accidentes (Steenbekkers \& Molenbroek, 1990). Para el caso de los alumnos, se ha reportado la importancia que tiene considerar el tamaño corporal de ellos para determinar las dimensiones y diseño del mobiliario educativo a fin de evitar problemas musculo-esqueléticos (Castellucci et al., 2010; Gouvali \& Boudolos, 2006; Molenbroek et al., 2003; Panagiotopoulou et al., 2004). Al respecto el uso de mobiliario inadecuado causa dolor en espalda, hombros y cuello y potenciales lesiones en el sistema musculoesquelético (Grimmer \&
Williams, 2004). En Chile la información antropométrica objetiva para dichos grupos etarios está orientada a observar el crecimiento y desarrollo infantil, por lo que no es adecuada para el proceso de diseño de productos (Ávila Chaurand et al.).

El mobiliario que se define como normativo en las salas de actividades de los niños que atiende la Junta Nacional de Jardines Infantiles (JUNJI) es un diseño que data de los inicios de la institución en 1970, sin un estudio antropométrico conocido para definir sus dimensiones. No se ha considerado que la morfoestructura humana sufre modificaciones a lo menos cada diez años.

\footnotetext{
Facultad de Diseño, Universidad del Desarrollo, Santiago, Chile; Escuela de Kinesiología, Facultad de Medicina, Universidad Mayor, Santiago, Chile.

** Laboratorio de Antropología Física y Anatomía Humana, Facultad de Ciencias, Instituto de Biología, Pontificia Universidad Católica de Valparaíso, Chile.

**** Instituto de Seguridad del Trabajo. Escuela de Kinesiología, Facultad de Medicina, Universidad Mayor, Chile.

El "Estudio Ergonómico para determinar las dimensiones del Mobiliario JUNJI" fue financiado por la Junta Nacional de Jardines Infantiles bajo Contrato RS 015/2942 del 27 de diciembre de 2007. Inscripción de Propiedad Intelectual a nombre de Junta Nacional de Jardines Infantiles bajo No 198.835 del 25/ $11 / 2010$ y que en fecha 28/03/2011 JUNJI autoriza a la autora principal del presente trabajo la reproducción total o parcial de la obra.

El estudio además fue apoyado por la Escuela de Kinesiología de la Universidad Mayor sede Santiago, con el patrocinio del equipamiento antropométrico.
} 
En el año 2002 se realizó un estudio preliminar en la comuna de La Pintana en Santiago a objeto de verificar la correspondencia entre la antropometría de los párvulos y el mobiliario que utilizan, recomendando incrementar a 3 los tamaños de mobiliario para favorecer condiciones posturales adecuadas. Adicionalmente explicita la necesidad de realizar un estudio de mayor envergadura (Córdova et al., 2002).

Ante la necesidad de determinar las dimensiones del mobiliario normativo, en 2007 JUNJI encarga un estudio ergonómico que consideró un levantamiento antropométrico para conocer el tamaño corporal de los niños que atiende JUNJI.

\section{MATERIAL Y MÉTODO}

Se realizó un muestreo unietápico por conglomerados sin afijación proporcional. Los jardines se seleccionaron por los que tenían mayor cantidad de matrícula, debido a que se medían todos los niños encontrados en el jardín el día de medición, previo consentimiento informado de los padres y equipo educativo del Jardín y las autoridades regionales de la institución.

Los niños fueron medidos descalzos, en camiseta y ropa interior, para lo cual educadoras y algunas madres asistieron ese día en el apoyo de la actividad.

Sujetos. Se obtuvieron datos de 2338 niños (1129 niñas y 1209 niños) de educación parvularia de Jardines Infantiles Clásicos de la JUNJI, en las ciudades de Iquique y Alto Hospicio (I Región), Temuco, Padre las Casas, Pucón, Victoria, Loncoche (IX Región), Osorno, Puerto Montt, Ancud (X Región), Punta Arenas (XII Región) y las comunas de Huechuraba, La Granja, La Pintana, Puente Alto, Conchalí, La Florida y Cerro Navia en la Región Metroplitana. Las ciudades medidas fuera de la Región Metropolitana se determinaron a objeto de "cubrir" posibles diferencias morfológicas a causa de diversidad multicultural.

La Institución atiende infantes desde el fin del postnatal al ingreso a la escuela. Si bien actualmente el postnatal es hasta la semana doce de nacido y los niños entran a escuelas a los 5 años, en la fecha de la evaluación se atendía a párvulos desde los 84 días de nacido a los 6 años de edad que se agruparon en 6 Tramos de edad (Tabla I).

Equipos. Para las evaluaciones en terreno se utilizó el Kit Gaucho Pro "Mercosur", fabricado en Argentina bajo licencia de Rosscraft Canadá para su comercialización en el Continente Americano. Del kit antropométrico se utilizaron los instrumentos: Campbell 20 (Antropómetro largo), Segmentómetro. Adicionalmente se utilizó un tallímetro para estatura.

Se construyó un prisma de $13 \mathrm{~cm}$ x $17 \mathrm{~cm}$ x $22 \mathrm{~cm}$ que, en conjunto con las sillas existentes en los jardines, modificaba las alturas de altura poplítea de los párvulos, logrando la postura sentada de evaluación en todos los casos (Fig. 1).

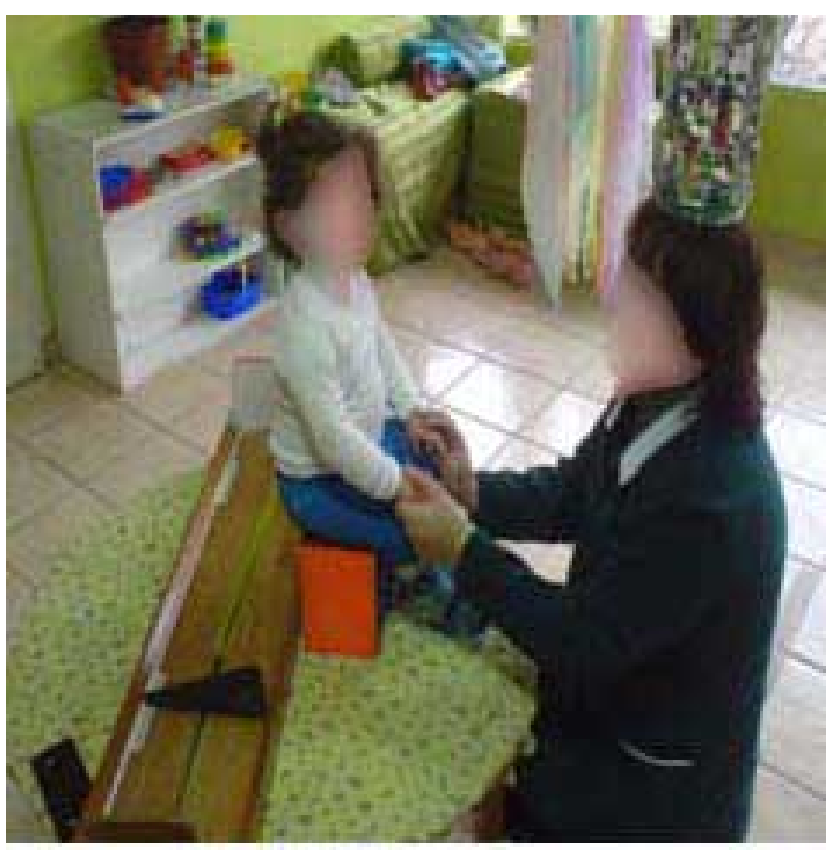

Figura 1. Menor sentada en prisma para mediciones en postura sentada. Educadora de apoyo para el manejo de los párvulos.

Los instrumentos se ubicaron dentro de una habitación seleccionada con 3 estaciones para mediciones.

- Estación para evaluar en postura de pie: Se habilitó una cinta métrica metálica sobre una muralla limpia sin guardapolvos ni imperfecciones en la superficie del muro ni en la del piso ni elementos que entorpezcan la postura del menor evaluado. La cinta métrica se puso perpendicular al suelo (uso de escuadra más dos puntos equidistantes de una horizontal para evitar cualquier inclinación de la huincha con respecto al piso).

- Estación alternativa para evaluar a los niños menores fueron evaluados acostados sobre podómetro ubicado sobre una mesa.

- Estación para evaluar en postura sentado: Sobre una mesa se ubicó un asiento seleccionado dentro de los que estaban en el jardín, complementando con el poliedro con el fin de mantener una postura sedente. 
Tabla I: Grupos etarios de la muestra.

\begin{tabular}{cc}
\hline 3 a 9 meses & Sala Cuna \\
10 a 15 meses & Sala Cuna \\
16 a 24 meses & Sala Cuna \\
25 a 36 meses & Medio \\
37 a 48 meses & Medio \\
49 a 72 meses & Transición \\
\hline
\end{tabular}

Variables consideradas. A objeto de conocer las dimensiones antropométricas que se requieren para referenciar el mobiliario normativo que usa JUNJI se determinaron 15 parámetros a evaluar, usualmente utilizados para estudios similares (Ávila-Chaurand et al., 2001) al que se sumaron 2 dimensiones más: la Altura de Horquilla para referenciar altura de barandas seguras de cunas y el Ancho de Codos Amplio que permite definir un ancho cómodo de uso en superficies de trabajo.

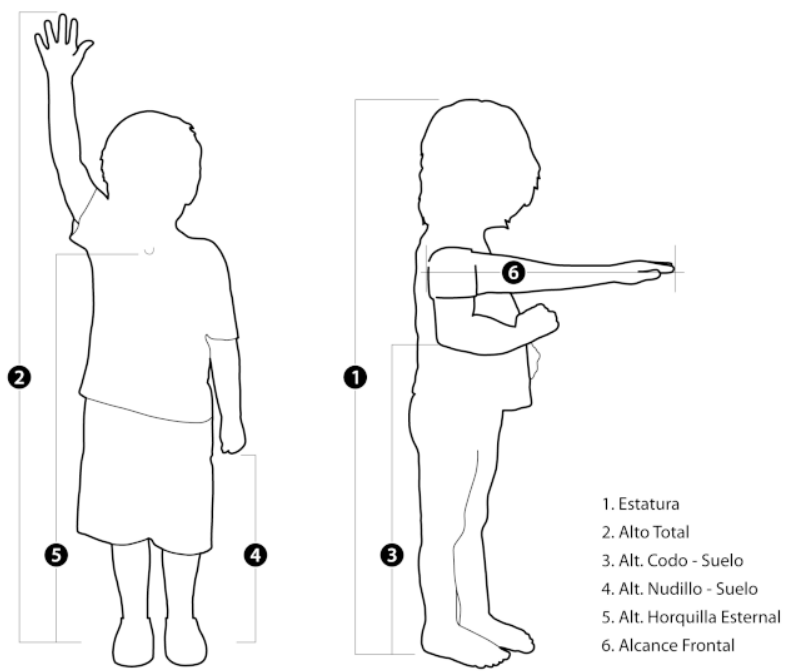

Fig. 2. Variables antropométricas en postura de pie.
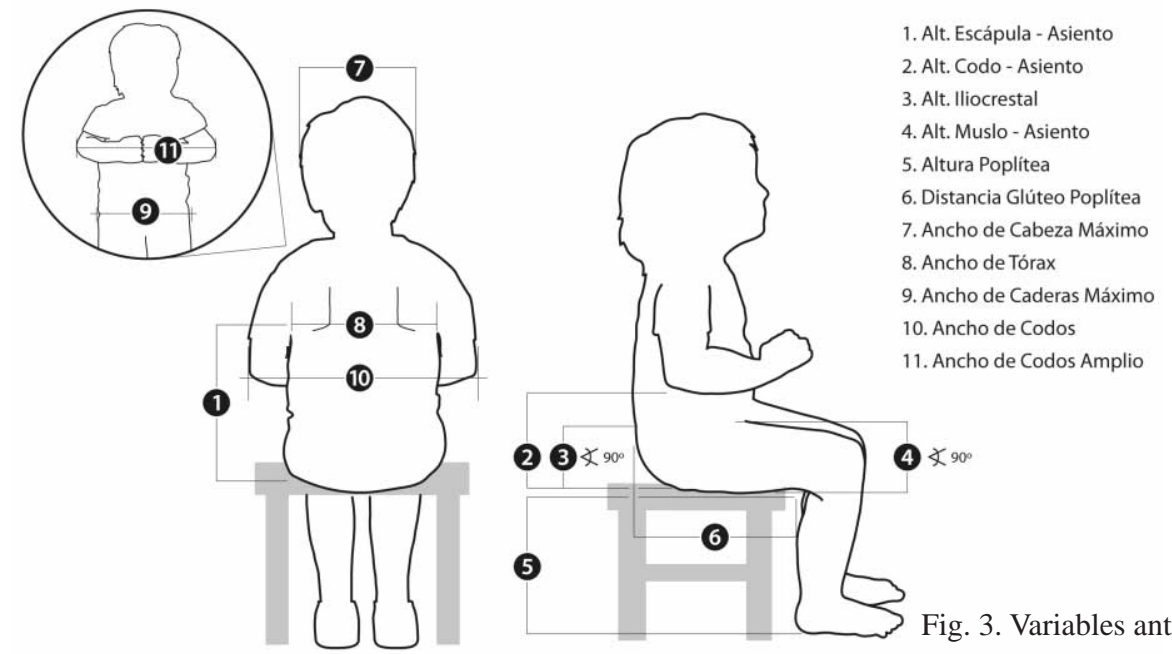

Fig. 3. Variables antropométricas en postura sentado.

Para la evaluación se consideran 3 posturas de referencia:

- Postura de Pie: apoyado contra el muro de medición, hombros relajados, cabeza orientada en plano metaorbitario (de Frankfurt)

- Postura decúbito dorsal: Acostado con pies apoyados en la base del podómetro y cabeza orientada en plano metaorbitario respecto del plano de trabajo, para los menores de 9 meses de edad. Cabe señalar que no todos los infantes del tramo 1 pudieron evaluarse en todas las variables ya era difícil evaluarlas sin forzar la postura artificialmente.

- Postura sentado: Espalda erguida con triple flexión de $90^{\circ}$ para la articulación de coxofemoral (cadera) y femorotibial (rodilla) dejando la articulación tibioastragalina (tobillo) en posición neutra y ambos pies apoyados completamente en la mesa de base. Para lograr la misma posición en los párvulos más pequeños, se ubicó el prisma sobre una mesa para sentarles y solucionar la diferencia que existe entre las dimensiones de los miembros inferiores de los menores y permitiendo mantener una posición recta de la rodilla $\left(90^{\circ}\right.$ de flexión).

Personal Levantamiento Antropométrico. El trabajo se realizó durante el año 2008 por equipos constituidos por el evaluador (Kinesiólogo capacitado en Antropometría) y con un asistente (usualmente Nutricionista del equipo regional de JUNJI), quien es el encargado de anotar los datos en planilla Excel previa confirmación oral de cada cifra. El ordenamiento de las columnas está definido por la coreografía de medición, desde superior a inferior en postura de pie y después sentado. Se cuenta con la ayuda de educadoras de párvulos del mismo Jardín para la entrega de los datos generales por infante (nombre con sus dos apellidos, cédula de identidad, sexo y fecha de nacimiento) y el apoyo de mamás de los mismos niños y niñas para mantener el orden, desvestir y vestir.
1. Alt. Escápula - Asiento

8. Ancho de Tórax

de Caderas Máximo

10. Ancho de Codos
El análisis de los datos fue realizado en planillas Excel de Microsoft Office 2007. 
RESULTADOS. Los resultados de este estudio son presentados de manera que sirvan a determinar las dimensiones para el diseño de equipamiento para la primera infancia. Separados por sexo y tramo de edad, las Tablas muestran Promedio y Desviación Estándar y percentiles 5 y 95 para la toma de decisiones de diseño.

La Tabla II presenta los resultados de la evaluación en Postura de Pie. La Tabla III exhibe los resultados de la evaluación en postura sentado.

Tabla II. Medidas antropométricas de párvulos chilenos en postura de pie

\begin{tabular}{|c|c|c|c|c|c|c|c|c|c|c|}
\hline \multirow{2}{*}{$\begin{array}{l}\text { ESTATURA } \\
\text { Grupo etáreo }\end{array}$} & \multicolumn{4}{|c|}{ Sexo Masculino } & \multirow[b]{2}{*}{$\mathbf{n}$} & \multicolumn{5}{|c|}{ Sexo Femenino } \\
\hline & Promedio & DE & 5 per & 95 per & & Promedio & DE & 5 per & 95 per & $\mathbf{n}$ \\
\hline Hasta 9 meses & 70,99 & 5,64 & 64,46 & 80,98 & 23 & 64,04 & 3,63 & 59,38 & 69,2 & 12 \\
\hline 10 a 15 meses & 75,93 & 5,47 & 70,46 & 82,7 & 63 & 72,68 & 3,28 & 67,04 & 79,06 & 48 \\
\hline 16 a 24 meses & 82,57 & 4,16 & 75,53 & 90,18 & 146 & 81,17 & 3,99 & 74,72 & 87,39 & 123 \\
\hline 25 a 36 meses & 91,3 & 4,27 & 84,5 & 99 & 260 & 90,08 & 3,93 & 83,5 & 96,17 & 227 \\
\hline 37 a 48 meses & 98,61 & 4,18 & 92,45 & 106,95 & 351 & 97,39 & 4,23 & 90,71 & 104,10 & 348 \\
\hline 49 meses y más & 105,31 & 4,39 & 98,31 & 112,7 & 362 & 104,45 & 5,19 & 96,6 & 113,17 & 369 \\
\hline ALTO TOTAL & \multicolumn{4}{|c|}{ Sexo Masculino } & & \multicolumn{5}{|c|}{ Sexo Femenino } \\
\hline Grupo etáreo & Promedio & DE & 5 per & 95 per & $\mathbf{n}$ & Promedio & DE & 5 per & 95 per & $\mathbf{n}$ \\
\hline Hasta 9 meses & 82,13 & 7,8 & 74,1 & 95,3 & 21 & 72,09 & 5,51 & 65,31 & 79,78 & 12 \\
\hline 10 a 15 meses & 87,99 & 7,32 & 79,08 & 96,2 & 61 & 84,27 & 4,75 & 76,06 & 90,84 & 49 \\
\hline 16 a 24 meses & 96,95 & 5,48 & 88,43 & 106,33 & 146 & 95,13 & 5,61 & 86,28 & 103,1 & 124 \\
\hline 25 a 36 meses & 108,73 & 5,84 & 100 & 118,5 & 258 & 106,69 & 5,91 & 98,33 & 117,19 & 226 \\
\hline 37 a 48 meses & 117,73 & 5,52 & 110 & 127,6 & 351 & 116,31 & 5,69 & 107,02 & 124,9 & 345 \\
\hline 49 meses y más & 127,22 & 6,07 & 117,41 & 136,59 & 363 & 126 & 7,44 & 115,23 & 137,2 & 367 \\
\hline $\begin{array}{l}\text { ALTURA } \\
\text { CODO-SUELO }\end{array}$ & \multicolumn{4}{|c|}{ Sexo Masculino } & & \multicolumn{5}{|c|}{ Sexo Femenino } \\
\hline Grupo etáreo & Promedio & DE & 5 per & 95 per & $\mathbf{n}$ & Promedio & DE & 5 per & 95 per & $\mathbf{n}$ \\
\hline Hasta 9 meses & 41,77 & 4,1 & 37,51 & 49,55 & 23 & 36,52 & 2,45 & 33,72 & 39,97 & 12 \\
\hline 10 a 15 meses & 43,82 & 3,73 & 40,42 & 47,9 & 61 & 42,51 & 2,42 & 38,42 & 47,83 & 49 \\
\hline 16 a 24 meses & 48,51 & 3,16 & 43,65 & 53,83 & 146 & 47,65 & 3,03 & 42,94 & 52,44 & 125 \\
\hline 25 a 36 meses & 53,89 & 3,11 & 48,87 & 59,36 & 258 & 52,92 & 3,1 & 48,84 & 58,53 & 225 \\
\hline 37 a 48 meses & 57,2 & 3,08 & 52,55 & 62 & 351 & 56,92 & 3,82 & 52,04 & 61,33 & 348 \\
\hline 49 meses y más & 61,66 & 3,28 & 56,22 & 66,7 & 365 & 61,36 & 3,82 & 55,4 & 67,7 & 368 \\
\hline $\begin{array}{l}\text { ALTURA } \\
\text { NUDILLO-SUELO }\end{array}$ & \multicolumn{4}{|c|}{ Sexo Masculino } & & \multicolumn{5}{|c|}{ Sexo Femenino } \\
\hline Grupo etáreo & Promedio & DE & 5 per & 95 per & n & Promedio & DE & 5 per & 95 per & $\mathbf{n}$ \\
\hline Hasta 9 meses & 30,1 & 3,44 & 26,16 & 36,59 & 23 & 26,83 & 2,04 & 24,28 & 29,53 & 12 \\
\hline 10 a 15 meses & 31,65 & 2,92 & 28,4 & 35,2 & 61 & 31 & 2,13 & 27,56 & 35,2 & 49 \\
\hline 16 a 24 meses & 35,06 & 2,67 & 31,3 & 39,1 & 146 & 34,77 & 2,78 & 30,46 & 39,1 & 125 \\
\hline 25 a 36 meses & 38,49 & 2,66 & 34,4 & 43,06 & 260 & 38,07 & 3,48 & 34,2 & 43,24 & 226 \\
\hline 37 a 48 meses & 40,13 & 2,29 & 36,6 & 44,2 & 351 & 39,87 & 2,68 & 36,44 & 43,63 & 348 \\
\hline 49 meses y más & 42,91 & 3,6 & 38,34 & 46,7 & 365 & 42,92 & 2,82 & 38,5 & 47,97 & 368 \\
\hline \multicolumn{6}{|c|}{$\begin{array}{l}\text { ALTURA } \\
\text { HORQUILLA ESTERNAL }\end{array}$} & \multicolumn{5}{|c|}{ Sexo Femenino } \\
\hline Grupo etáreo & Promedio & DE & 5 per & 95 per & n & Promedio & DE & 5 per & 95 per & $\mathbf{n}$ \\
\hline Hasta 9 meses & 53,37 & 5,23 & 47,4 & 63,04 & 23 & 47,67 & 2,79 & 44,32 & 51,82 & 12 \\
\hline 10 a 15 meses & 58,46 & 7,5 & 53,09 & 63,2 & 61 & 55,46 & 2,77 & 49,72 & 60,29 & 49 \\
\hline 16 a 24 meses & 63,25 & 4,64 & 57,74 & 69,56 & 145 & 62,54 & 3,49 & 57,1 & 67,56 & 125 \\
\hline 25 a 36 meses & 70,33 & 4,71 & 63,8 & 76,7 & 260 & 69,77 & 3,77 & 64,73 & 75,87 & 226 \\
\hline 37 a 48 meses & 76,36 & 4,46 & 70,55 & 83,31 & 350 & 75,47 & 4,25 & 69,5 & 81,63 & 348 \\
\hline 49 meses y más & 82,04 & 4,84 & 75,16 & 89,09 & 365 & 81,89 & 4,92 & 74,6 & 89,57 & 367 \\
\hline \multicolumn{2}{|c|}{ ALCANCE FRONTAL } & \multicolumn{4}{|c|}{ Sexo Masculino } & \multicolumn{5}{|c|}{ Sexo Femenino } \\
\hline Grupo etáreo & Promedio & DE & 5 per & 95 per & $\mathbf{n}$ & Promedio & DE & 5 per & 95 per & $\mathbf{n}$ \\
\hline Hasta 9 meses & 32,55 & 2,47 & 29,14 & 36,67 & 23 & 28,72 & 2,06 & 25,78 & 31,35 & 12 \\
\hline 10 a 15 meses & 33,96 & 4,25 & 29,8 & 37,3 & 61 & 32,65 & 1,73 & 30,26 & 37,24 & 49 \\
\hline 16 a 24 meses & 36,3 & 2,28 & 33,2 & 40,18 & 145 & 35,57 & 1,95 & 32,64 & 38,58 & 125 \\
\hline 25 a 36 meses & 40,55 & 4,05 & 36,5 & 44,74 & 260 & 39,96 & 2,7 & 35,93 & 44,61 & 226 \\
\hline 37 a 48 meses & 45,17 & 2,79 & 41,25 & 50,65 & 351 & 44,3 & 2,79 & 40,27 & 48,87 & 348 \\
\hline 49meses y más & 48,89 & 3,19 & 44,3 & 54,1 & 365 & 48,23 & 3,55 & 42,74 & 53,47 & 368 \\
\hline
\end{tabular}


Tabla III. Medidas antropométricas de párvulos chilenos en postura sentado.

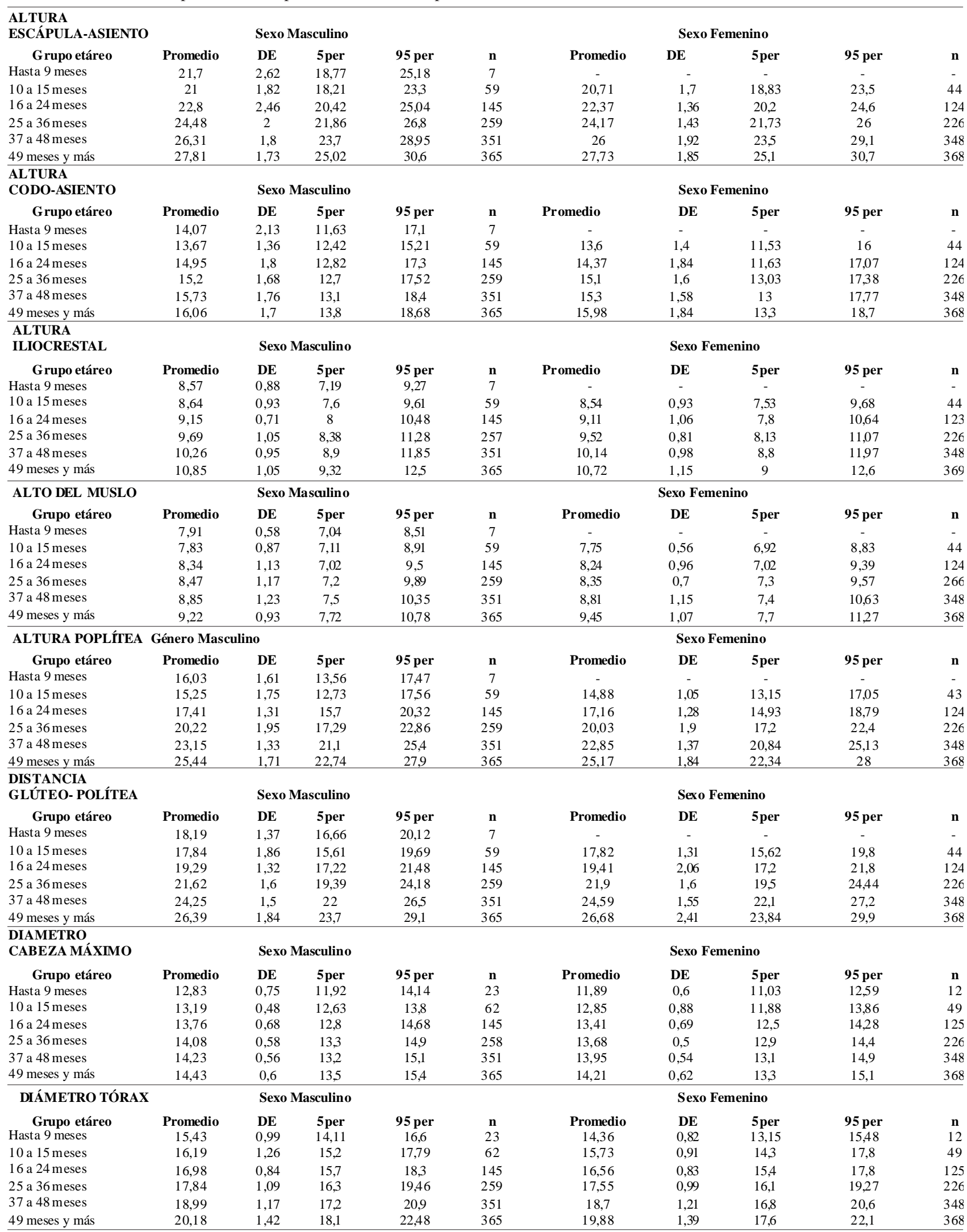




\begin{tabular}{|c|c|c|c|c|c|c|c|c|c|c|}
\hline \multicolumn{2}{|c|}{ ANCHURA DE CADERAS } & \multicolumn{3}{|c|}{ Sexo Masculino } & \multicolumn{6}{|c|}{ Sexo Femenino } \\
\hline Grupo etáreo & Promedio & DE & 5 per & 95 per & $\mathbf{n}$ & Promedio & DE & 5 per & 95 per & $\mathbf{n}$ \\
\hline Hasta 9 meses & 18,47 & 1,54 & 16,38 & 20,91 & 20 & 17,49 & 1,27 & 16,15 & 19,6 & 11 \\
\hline 10 a 15 meses & 19,21 & 1,9 & 17,03 & 21,55 & 59 & 18,61 & 1,17 & 16,95 & 21,83 & 46 \\
\hline 16 a 24 meses & 19,83 & 1,41 & 17,33 & 22,17 & 144 & 19,8 & 1,43 & 17,42 & 21,8 & 124 \\
\hline 25 a 36 meses & 20,63 & 1,67 & 18,3 & 23,5 & 259 & 20,78 & 1,48 & 18,53 & 23,07 & 226 \\
\hline 37 a 48 meses & 21,75 & 1,69 & 19,2 & 24,9 & 351 & 22,07 & 1,68 & 19,64 & 25,07 & 348 \\
\hline 49 meses y más & 22,57 & 1,82 & 20,1 & 26,1 & 362 & 23,36 & 1,93 & 20,3 & 26,8 & 368 \\
\hline \multicolumn{2}{|c|}{ ANCHO ENTRE CODOS } & \multicolumn{3}{|c|}{ Sexo Masculino } & \multicolumn{6}{|c|}{ Sexo Femenino } \\
\hline Grupo etáreo & Promedio & DE & 5 per & 95 per & $\mathbf{N}$ & Promedio & DE & 5 per & 95 per & $\mathbf{n}$ \\
\hline Hasta 9 meses & 25,07 & 2,4 & 22,21 & 27,9 & 23 & 23,32 & 1,62 & 21,8 & 26,35 & 12 \\
\hline 10 a 15 meses & 25,68 & 2 & 23,96 & 28,9 & 61 & 25,01 & 1,83 & 22,69 & 28,9 & 48 \\
\hline 16 a 24 meses & 26,49 & 1,76 & 24,23 & 29,29 & 144 & 25,94 & 1,65 & 23,41 & 28,89 & 123 \\
\hline 25 a 36 meses & 27,17 & 1,96 & 24,08 & 30,64 & 259 & 26,85 & 1,85 & 23,95 & 29,95 & 226 \\
\hline 37 a 48 meses & 28,47 & 2,1 & 25,4 & 31,85 & 348 & 27,99 & 2,12 & 24,77 & 31,3 & 348 \\
\hline 49 meses y más & 29,31 & 2,18 & 26,02 & 32,06 & 365 & 29,4 & 2,36 & 25,84 & 33,2 & 368 \\
\hline \multicolumn{5}{|l|}{$\begin{array}{l}\text { ANCHO ENTRE } \\
\text { CODOS AMPLIO }\end{array}$} & \multicolumn{6}{|c|}{ Sexo Femenino } \\
\hline Grupo etáreo & Promedio & DE & 5 per & 95 per & $\mathbf{n}$ & Promedio & DE & 5 per & 95 per & $\mathbf{n}$ \\
\hline Hasta 9 meses & 30,61 & 3,41 & 25,49 & 33,53 & 8 & - & - & - & - & - \\
\hline 10 a 15 meses & 30,74 & 2,58 & 27,73 & 33,42 & 58 & 29,03 & 1,91 & 25,62 & 35,1 & 43 \\
\hline 16 a 24 meses & 33,58 & 2,02 & 30,32 & 37,17 & 144 & 32,72 & 1,78 & 29,84 & 35,6 & 123 \\
\hline 25 a 36 meses & 37,41 & 2,31 & 33,79 & 40,78 & 259 & 36,53 & 2,02 & 33,24 & 39,68 & 225 \\
\hline 37 a 48 meses & 40,74 & 2,06 & 37,65 & 44,1 & 351 & 39,77 & 2,39 & 36,4 & 43,27 & 348 \\
\hline 49 meses y más & 43,61 & 2,21 & 40,22 & 47,39 & 364 & 42,67 & 2,5 & 38,9 & 46,83 & 368 \\
\hline
\end{tabular}

\section{DISCUSIÓN}

Hay consenso internacional acerca de la relación que debe existir entre el mobiliario educativo que se utiliza en las salas y la antropometría de los usuarios (Castellucci et al.; Molenbroek et al.; Molenbroek \& Ramaekers, 1996). En 1998 en Chile se realiza un estudio antropométrico en escolares (MINEDUC et al., 2001), el cual no alcanza a cubrir a los infantes en edad preescolar.

Estos y otros estudios suelen referenciar normas internacionales para mobiliario educativo (CEN - European Commitee for Standarization, 2006; ISO 597O, 1979). A nivel nacional el mobiliario escolar también se basa en el mencionado estudio a escolares realizado en conjunto por el Ministerio de Educación y UNESCO para determinar las 19 normas estandarizadas en la materia, todas bajo el título de "Mobiliario Escolar". Ante la falta de información en materia de antropometría de párvulos chilenos, las normas nacionales se refieren a materias tales como Norma de Muestreo para el Control de Calidad (NCh2619) (INN, 2003a), y pruebas de simulación de solicitaciones mecánicas (NCH2656; NCh2609; NCh2226, INN, 2003b).

En un estudio preliminar aplicado a párvulos en la comuna de La Pintana (Córdova et al.), se identifica una discrepancia importante entre las dimensiones antropométricas de los niños y el mobiliario existente, así como la necesidad de realizar un estudio más amplio que determine los requerimientos dimensionales para el mobiliario de los Jardines Infantiles JUNJI, así como el que se establece como normativo para los jardines que reciben recursos del Estado (JUNJI, 2011).
Las Tablas antropométricas del presente documento son el resultado de un estudio mayor, realizado en diversas comunas de distintas ciudades del país y fueron entregados dentro de un informe con las recomendaciones de modificación de dimensiones para cada uno de los muebles normativos de la Institución (Rojas \& Ilardi, 2009).

En los últimos años las políticas públicas chilenas han venido desarrollando sostenidamente una serie de acciones para promover la calidad en Educación Parvularia. Los resultados del presente estudio son parte de las iniciativas de JUNJI para promover la calidad de la Educación Parvularia nacional, particularmente en lo referido al Ambiente Físico de Aprendizaje (Valderrama et al., 2010), a objeto de mejorar las condiciones para el aprendizaje, comodidad, seguridad y salud de los párvulos.

Al respecto, la Escala ECERS-R (Harms et al., 1998) declara como "bueno" cuando la mayoría del mobiliario es adecuado al tamaño infantil, explicitando en la versión en español de manera simple y observable, la postura de referencia para la postura sentado (pies apoyados en el suelo al estar sentado, codos encima de la mesa, espacio libre bajo la mesa que no obstruya las extremidades inferiores), también expresada por MINEDUC-UNESCO (MINEDUC et $a l$. ), entre otros.

El levantamiento antropométrico cuyas Tablas se expresan en este artículo, permiten acercarse a conocer la morfoestructura de los párvulos chilenos, como parte del 
estudio ergonómico para determinar las medidas del mobiliario y la consecuente actualización de la normativa nacional en la material, favoreciendo mejorar las condiciones de comodidad, seguridad y salud de los párvulos, así como posibilitar algún incremento en el puntaje en la Subescala de Ambiente Físico y Mobiliario ECERS-R para los Jardines Infantiles JUNJI u otros que reciben aporte del Estado

\section{AGRADECIMIENTOS}

Junta Nacional de Jardines Infantiles. Al Departamento Técnico de Dirección Nacional por su constancia y colaboración técnico educativa espacializada. Agradecer a las Direcciones Regionales en las gestiones y coordinación, así como el apoyo durante las visitas de evaluación. Agradecer a los equipos directivos y educativos de los Jardines Infantiles muestrales su disposición y colaboración. Agradecer a las comunidades educativas de los jardines, quienes organizaron un grupo de mamás que fue a apoyar la evaluación en sala, de manera que el equipo de investigación recibió un apoyo invaluable para el trabajo en terreno y manejo infantil.

Escuela de Kinesiología, Facultad de Medicina Universidad Mayor, por su apoyo al facilitar el instrumental de antropometría que permitió la evaluación en terreno y particularmente la colaboración en revisión del análisis de datos por el Dr. Oscar Urrejola, Profesor de la Escuela de Kinesiología y Coordinador del Área de Gestión de la Escuela de Terapia Física, de la U. Mayor.

Laboratorio de Antropología Física y Anatomía Humana de la Pontificia Universidad Católica de Valparaíso, por la constancia y colaboración en el trabajo. Por otro lado agradecer a la Dirección de Investigación, Vice-rectoría de Investigación y Estudios Avanzados e Instituto de Biología de la Facultad de Ciencias. Pontificia Universidad Católica de Valparaíso. Chile, por su constante apoyo en materia investigativa.

ROJAS, C. J.; ALMAGIÀ, F. A. A. \& ILARDI, J. S. Anthropometric study of infants in the Chilean public education system for furniture design. Int. J. Morphol., 31(1):189-196, 2013.

SUMMARY: To update the design of furniture used by Chilean preschool children in the public education system since 1970, a Ergonomic study is carried out in order to establish criteria and dimensions to propose a standard for the design of new furniture. This paper presents the results of an anthropometric survey conducted on 2338 children from 3 months to 6 years of age. in preschools of the Junta Nacional de Jardines Infantiles (JUNJI) in Regions I, IX, X, XII and the Metropolitan region. While a study of the variables of height and weight recorded in the Information Management System of the Institution for children of classic urban preschools showed no significant differences between geographical areas, the regions were selected considering intercultural diversity by direct ancestors of foreign origin and / or indigenous peoples in case the proportionality of the segments manifested differences, even if height and weight were similar. Anthropometric parameters were measured 19 for the development of preschool furniture ergonomics standard to which a variable to guide the use of a wide work surface was added. Considering the results of the sample considered, we can state that changes are necessary in the dimensions of the furniture in use. We found a significant discrepancy between the anthropometric dimensions of children and the existing furniture. Anthropometric tables are proposed for children from 3 to 72 months of age, which would indicate that the furniture in use is not the right size to ensure comfort and convenience.

KEY WORDS: Infants; Anthropometric; Ergonomics; Preschool furniture.

\section{REFERENCIAS BIBLIOGRAFICAS}

Ávila Chaurand, R.; Prado León, L. \& González Muñoz, E. Dimensiones Antropométricas de la Población Latinoamericana: México, Cuba, Colombia, Chile. Guadalajara, Universidad de Guadalajara, 2001.

Castellucci, H. I.; Arezes, P. M. \& Viviani, C. A. Mismatch between classroom furniture and anthropometric measures in Chilean schools. Appl. Ergon., 41(4):563-8, 2010.

CEN (European Commitee for Standardization). Furniture : chairs and tables for educational institutions. Part 1, Functional dimensions. BS EN 1729-1 : 2006. London, European Commitee for Standardization, 2006.
Córdova, V. P.; Muñoz, M. M.; Aguirre, R. L.; Celedón, O. A. \& Almagia, F. A. Evaluación del Mobiliario Utilizado por Párvulos JUNJI Basada en un Estudio de Antropometría. Bol. Cient. Asoc. Chil. Segur., 4(8):42-9, 2002.

Gouvali, M. K. \& Boudolos, K. Match between school furniture dimensions and children's anthropometry. Appl. Ergon., 37(6):765-73, 2006.

Grimmer, K. \& Williams, M. Gender-age environmental associates of adolescent low back pain. Appl. Ergon., 31(4):343-60, 2000.

Harms, T.; Clifford, D. \& Cryer, D. ECERS-R. Early Childhood 
Environmental Rating Scale. New York, Teacher College Press, 1998.

INN (Instituto Nacional de Normalización). NCh2609.Of2002. Mobiliario escolar - Sillas para párvulos - Requisitos. Santigo, Instituto Nacional de Normalización, 2003a.

INN (Instituto Nacional de Normalización). NCh2619.Of2002, Mobiliario escolar - Muestreo. Santiago, Instituto Nacional de Normalización, 2003b.

ISO 597O. Furniture. Chairs and tables for educational institutions. Functional sizes. Geneva, International Organization for Standardization, 1979.

JUNJI. Resolución Excenta $\mathrm{N}^{\circ} 015 / 1645$. Instructivo del programa de transferencia de fondos desde JUNJI a entidades sin fines de lucro que creen, mantengan y/o administren jardines infantiles. Santiago, JUNJI, 2011.

MINEDUC; UNESCO; Universidad del Bío Bío \& Universidad de Concepción. Guía de Recomendaciones para el Diseño de Mobiliario Escolar (Proyecto Conjunto: MINEDUC/UNESCO (Código 916/CHI/11) ed.). Santiago, Ministerio de Educación, Gobierno de Chile, 2001.

Molenbroek, J. \& Ramaekers, Y. Anthropometric design of a size system for school furniture. Proceedings of the Annual Conference of the Ergonomics Society: Contemporary Ergonomics. London, Taylor \& Francis, 1996. pp.130-5.

Molenbroek, J. F.; Kroon-Ramaekers, Y. M. \& Snijders, C. J. Revision of the design of a standard for the dimensions of school furniture. Ergonomics, 46(7):681-94, 2003.

Panagiotopoulou, G.; Christoulas, K.; Papanckolaou, A. \& Mandroukas, K. Classroom furniture dimensions and anthropometric measures in primary school. Appl. Ergon., 35(2):121-8, 2004.

Rojas, J. \& Ilardi, J. S. Estudio ergonómico para determinar las dimensiones del mobiliario JUNJI. Santiago, JUNJI, 2009.

Steenbekkers, L. P. \& Molenbroek, J. F. Anthropometric data of children for non-specialist users. Ergonomics, 33(4):421-9, 1990 .

Valderrama, C.; Raczynski, D.; Morris, P. \& de la Fuente, L. Aporte de JUNJI a la generación de conocimiento para el mejoramiento de la política en materia de primera infancia. Sistematización de estudios 2006-2009. Asesorías para el desarrollo. Santiago, JUNJI, 2010.

\author{
Dirección para correspondencia: \\ Prof. Jimena Rojas Colvin \\ Facultad de Diseño \\ Universidad del Desarrollo \\ Av. Plaza 680, \\ San Carlos de Apoquindo \\ Las Condes, Santiago \\ CHILE
}

Fono 56 (02) 23279110

\author{
Email: jrojas@uc.cl \\ jimena.rojascolvin@gmail.com
}

Recibido : 07-08-2012

Aceptado: 10-01-2013 